IIIb. The rate of in-stent stenosis at final follow-up was $0 \%$.

Procedure-related complications occurred in 6 cases $(4.7 \%)$. There were five thrombotic complications (3.9\%), of which 2 were symptomatic $(1.6 \%)$. There was one case of common femoral pseudoaneurysm formation at the access site. There were no cases of procedure related mortality.

Conclusion Single stent assisted coiling is safe and effective for the treatment of wide-neck bifurcation aneurysms.

Disclosures D. Bageac: None. S. Gomez-Paz: None. J. Vranic: None. B. Gershon: None. W. Munoz Miranda: None. C. Stapleton: None. K. Fargen: None. A. Thomas: None. A. Patel: None. R. De Leacy: None.

\section{E-095 THE EMBOTRAP STENT RETRIEVER FOR TREATMENT OF ACUTE ISCHEMIC STROKE: IMPACT OF HOSPITAL PROCEDURAL VOLUME ON REAL WORLD OUTCOMES}

${ }^{1} S$ Ikeme*, , E Kottenmeier, ${ }^{1} \mathrm{~A}$ Khaled, ${ }^{2} \mathrm{~S} \mathrm{M},{ }^{3} \mathrm{R}$ Khanna. ${ }^{1}$ CERENOVUS Health Economics and Market Access, Johnson and Johnson, Irvine, CA; ${ }^{2}$ Mu Sigma Inc., Bangalore, INDIA; ${ }^{3}$ Medical Deviec Epidemiology and Real-World Data Sciences, Johnson and Johnson, New Brunswick, NJ

\subsection{6/neurintsurg-2021-SNIS.190}

Introduction The EmboTrap ${ }^{\oplus}$ Revascularization Device (CERENOVUS, Johnson \& Johnson Medical Devices, Irvine, CA, USA) is an innovative revascularization device with a dual layer design for thrombus retrieval. The objective of this study was to examine the impact of hospital mechanical thrombectomy (MT) volume on clinical outcomes among acute ischemic stroke (AIS) patients treated using the EmboTrap ${ }^{(8)}$ device.

Material and Methods Adult patients ( $\geq 18$ years), who underwent MT for AIS with the EmboTrap (alone or with another MT device) from July 2018-December 2019, were identified from the Premier Healthcare Database (PHD). The PHD is a nationwide hospital database, with more than 1,000 hospitals of varying size, location (urban/rural), geographic region, and teaching status. The influence of hospital MT volume (classified into quartiles based on MT volume in the six-month pre-index AIS admission period) on in-hospital mortality, length of stay (LOS), and rate of 30-day readmissions (all-cause and cardiovascular [CV]-related) was assessed. Generalized estimating equation regression was used to examine the relationship between MT volume and outcomes, adjusting for patient demographic and clinical characteristics, and provider characteristics. In addition, the use of other MT devices was controlled in study analyses. A logit link function was used for in-hospital mortality and readmission assessments and a log link function for LOS assessment. The distributions used were binomial distributions for in-hospital mortality and readmission assessments, and negative binomial distributions for LOS assessment.

Results The study included 400 patients (mean age 70.5 \pm 14.32 years) based on inclusion/exclusion criteria. Hospitals were classified into four volume categories: <63, 63-83, 84139 , and $>139$ MT procedures. Patients treated with EmboTrap (alone or with another MT device) in hospitals in the fourth MT volume quartile (>139 MT procedures) had lower odds of all-cause (odds ratio [OR] 0.22; 95\% confidence interval [CI] 0.05-0.96) and CV-related (OR 0.12; 95\% CI $0.02-0.65$ ) inpatient readmission as compared to patients treated with EmboTrap (alone or with another MT device) in hospitals in the first MT volume quartile (<63 procedures). No significant differences were observed in mortality and LOS.

Conclusions Hospital MT volume significantly influenced the rate of readmissions among AIS patients treated with the EmboTrap (alone or with another MT device) device. The lack of difference in mortality may also be reflective of limited statistical power to detect differences in study groups. Further research to understand the impact of hospital MT volume on patient outcomes, as well as identify the threshold volume that optimizes positive outcomes, could help inform future stroke care guidelines and contribute to improved treatment protocols in low-volume centers.

Disclosures S. Ikeme: None. E. Kottenmeier: None. A. Khaled: None. S. M: None. R. Khanna: None.

\section{E-096 MINIMALLY INVASIVE SURGERY FOR RESECTION OF VASCULAR LESIONS: A TECHNICAL REPORT ON THE USE OF THE BRAINPATH TUBULAR RETRACTOR SYSTEM IN PATIENTS WITH VARYING SUBCORTICAL VASCULAR LESIONS}

R Achey*, M Bain. Neurological Institute, Cleveland Clinic, Cleveland, $\mathrm{OH}$

\subsection{6/neurintsurg-2021-SNIS. 191}

Introduction/Purpose Vascular malformations in the subcortical and/or periventricular regions can cause devastating intraventricular/intracranial hemorrhages (IVH/ICH). These lesions are typically reached via open surgical techniques requiring significant cortical brain retraction to establish sufficient operative corridors for safe resection. The NICO BrainPath tubular retractor system offers a minimally invasive alternative for resection of deep-seated vascular lesions. Using a minimally invasive, trans-sulcal approach originally pioneered for tumor resection, this tubular retraction system has the potential to improve patient outcomes by reducing trauma to adjacent cortical tissue, utilizing smaller craniotomies, and reducing post-operative hospitalization times. Here, we report our experience using this minimally invasive approach for safe, and effective, complete resection of several subcortical vascular lesions.

Materials and Methods Patients were admitted to the neurosurgical service at our institution with varying subcortical vascular lesions. All participants (or family members) were consented for minimally invasive lesion resection using the NICO BrainPath $13.5 \mathrm{~mm}$ tubular retractor system. Magnetic resonance imaging (MRI) with stereotactic and DTI sequences was obtained prior to surgery. A trans-sulcal approach was used for tubular retractor insertion taking care to avoid important fiber tracks identified on pre-operative imaging. Post-operative imaging with MRI and/or angiogram was obtained to ensure complete lesion resection. Data was collected from six patients including age, initial diagnosis, technical success of surgery, follow-up imaging, and post-operative complications.

Results Seven patients ranging from 12 to 58 years old underwent minimally invasive BrainPath resection of deep-seated vascular lesions. Vascular lesions included: choroid plexus arteriovenous malformation (AVM) with resultant IVH, occipital periventricular AVM with resultant IVH, deep anterior communicating artery (ACA) mycotic aneurysm refractory to antibiotics, enlarging thalamic cavernous malformation, midbrain cavernoma with resultant IVH, gyrus rectus renal cell 


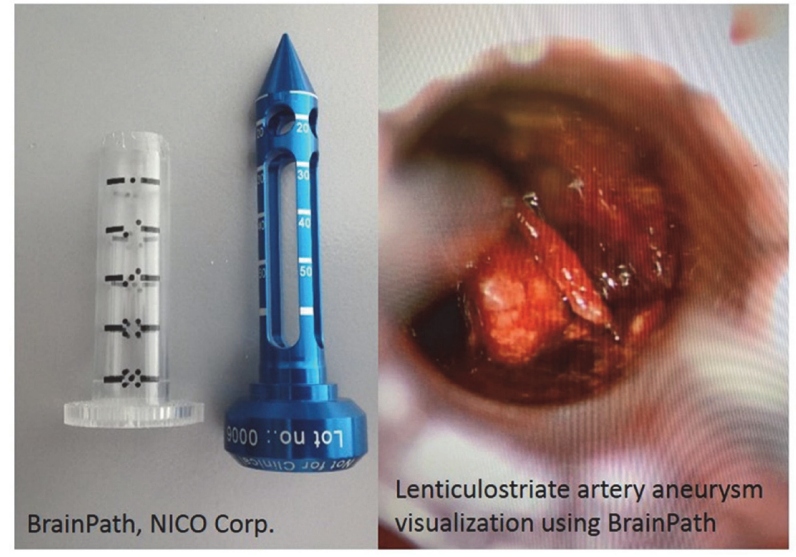

Abstract E-096 Figure 1

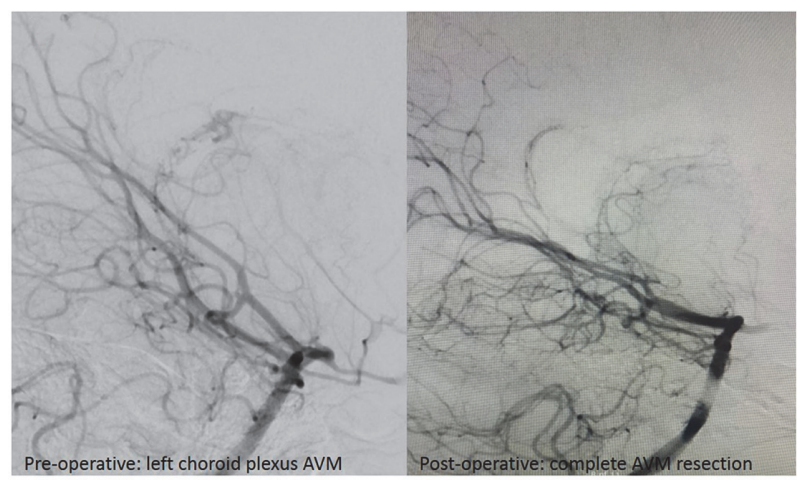

Abstract E-096 Figure 2

carcinoma metastasis with resultant IVH, and a lenticulostriate artery aneurysm with resultant basal ganglia hemorrhage. All patients had successful lesion resection, confirmed on postoperative imaging. There were no surgical complications.

Conclusions In this technical report, we demonstrate the effective use of a minimally invasive tubular retractor system for resection of various deep-seated vascular malformations and hemorrhagic masses. In all seven patients, lesions were successfully resected and no patient suffered surgical complications. This report shows the potential for minimally invasive techniques to adequately treat a wide variety of subcortical vascular lesions. This technique could revolutionize common approaches to open cerebrovascular surgery by mitigating neurological consequences of parenchymal insult relating to surgical retraction. Further research will determine whether minimally invasive techniques significantly improve patient outcomes.

Disclosures R. Achey: None. M. Bain: 2; C; Stryker, Cerenovus, Microvention, Rebound Therapeutics.

\section{E-097 SIMULTANEOUS TRANSARTERIAL AND TRANSVENOUS CONTRAST INJECTION TO REVEAL THE CONNECTION POINT FOR TREATMENT OF CAROTID CAVERNOUS FISTULA-TECHNICAL NOTE}

C Rupareliya* , J Fraser, L Sheikhi. University of Kentucky, Lexington, KY

10.1136/neurintsurg-2021-SNIS.192
Introduction Accessing cavernous sinus (CS) via inferior petrosal sinus (IPS) may become difficult sometimes for the treatment of carotid-cavernous fistulas (CCFs). We report a technique for simultaneous transarterial and transvenous contrast injection resulting in well opacification of IPS and fistulous point resulting in a successful entry using microcatheter.

Material and Methods A 58-year-old male was referred to our academic medical center for three weeks of right eye pain, now complicated by redness, diplopia, and blurry vision. Magnetic Resonance Imaging (MRI) brain at the outside hospital revealed a hemorrhagic lesion in right parietotemporal region. Computerized tomography-angiogram (CTA) of the head revealed filling of cavernous sinus during an arterial phase suspicious for CCF.

Under general anesthesia, after accessing right common femoral artery, 4F berenstein catheter was advanced over 0.035 angled glide wire to the proximal right internal carotid artery. Contrast injected through ICA showed CS but not the IPS [figure 1(a)]. Through the left common femoral vein, synchro 2 soft microwire was advanced through echelon 10 microcatheter. Venous guide catheter was advanced into right internal jugular vein (IJV) and distal access catheter was placed into sigmoid jugular junction. Injected contrast revealed IPS, but not CS [figure 1(b)]. A subsequent simultaneous hand injection with the microcatheter within the IPS and the diagnostic catheter in the left ICA elucidated the arteriovenous

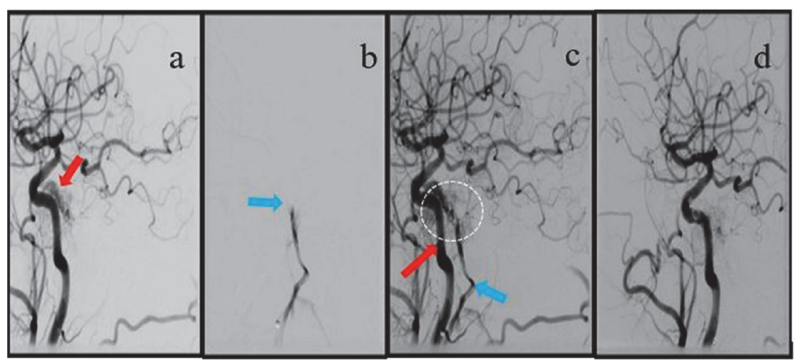

Abstract E-097 Figure 1 (a) There is flow into cavernous, but no visible flow through the IPS (b) Injection into right IJV opacifies half of IPS, but not the fistulous point or cavernous sinus (blue arrow) (c) Simultaneous injection into the right ICA (red arrow) and right IJV (blue arrow) pacifying the connection allowing subsequent navigation (white dashed circle) (d) Right iCA injection after successful coil embolization showing resolution

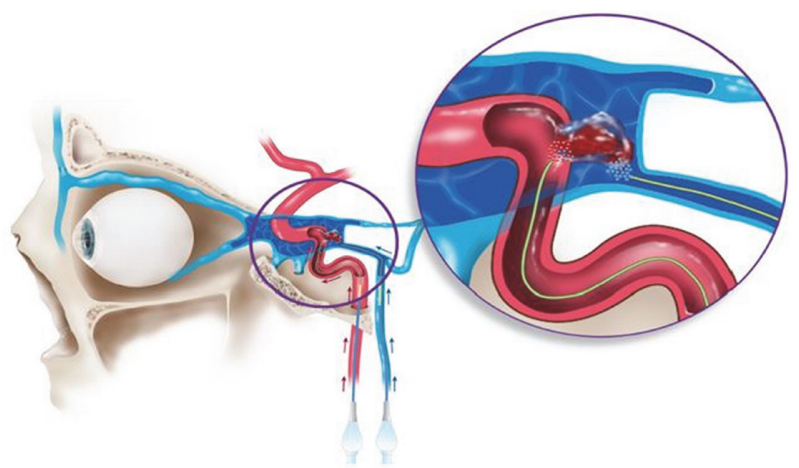

Abstract E-097 Figure 2 Illustration depicting simultaneous transarterial and transvenous contrast injection technique with point of connection magnified inside the circle 